\title{
DESARROLLO DE UN DISPOSITIVO AUTÓNOMO PARA EL MEJORAMIENTO DE LA CALIDAD DEL AGUA EN EL HUMEDAL CÓRDOBA (BOGOTÁ, COLOMBIA)
}

\section{DEVELOPMENT OF AN AUTONOMOUS DEVICE FOR WATER QUALITY IMPROVEMENT IN THE CÓRDOBA WETLAND (BOGOTA, COLOMBIA)}

\author{
BERMUDEZ, N. ${ }^{1}$; GALLEGOS, D. S. ${ }^{2}$; BOTELLO-SUÁREZ W. $A^{3}$ \\ ${ }^{1}$ Ing. Natalia Bermúdez Pinzón. Programa de Bioingeniería, \\ Facultad de Ingeniería, Universidad El Bosque. \\ nbermudezp@unbosque.edu.co \\ ${ }^{2}$ Ing. Diego Stiven Gallegos Bermúdez. Programa de \\ Bioingeniería, Facultad de Ingeniería, Universidad El Bosque. \\ dgallegos@unbosque.edu.co \\ ${ }^{3}$ PhD. Wilmar Alirio Botello Suárez. Profesor asociado, \\ Programa de Ingeniería Ambiental, Facultad de Ingeniería, \\ Universidad El Bosque. \\ wbotello@unbosque.edu.co
}

\author{
Entidad \\ Av. Cra. 9 No. 131 A - 02, Bogotá D.C, Colombia \\ PBX: (571) 6489000 \\ E-mail: wbotello@unbosque.edu.co
}

\begin{abstract}
Resumen
El humedal Córdoba (Bogotá, Colombia) es un sistema hídrico altamente afectado por la descarga de aguas residuales y actividades desarrolladas por la matriz urbana presente en sus inmediaciones. En este estudio se presenta el diseño, construcción y operación de un dispositivo portátil para la remoción simultánea de sólidos y nitrógeno amoniacal de aguas del humedal Córdoba, y que posibilita el incremento del oxígeno disuelto mediante aireación por gravedad. Para ello, hace uso de energía fotovoltaica y se incorpora un sistema de control de colmatación de filtros y obstrucción de manguera, con la finalidad de que presente un funcionamiento autónomo. Se consideraron variables tales como: pérdida de energía, diámetro y densidad de materiales filtrantes, tiempo de contacto, consumo eléctrico y peso, entre otros. Todos los diseños y ensayos de eficiencia fueron realizados conforme normas estandarizadas. Las dimensiones del dispositivo se estimaron en función del tiempo de retención hidráulica. Los resultados obtenidos permitieron medir la eficiencia del sistema, obteniendo remociones medias de $74,4 \%$ y $89,5 \%$ para amonio y sólidos totales, respectivamente, y un incremento de la concentración de oxígeno disuelto hasta de $45 \%$, tratando un caudal de $14 \mathrm{~L} / \mathrm{min}$ con una capacidad de trabajo autónomo
\end{abstract}


de $3.5 \mathrm{~h}$. Se concluye que el dispositivo diseñado constituye una alternativa promisoria para mejorar la calidad de las aguas del humedal Córdoba a nivel in situ, el cual puede ser potencialmente empleado para el tratamiento de aguas en otros sistemas hídricos de características similares.

Palabras clave: Aireación, amonio, energía fotovoltaica, eutrofización, sistema de control.

\begin{abstract}
The Córdoba wetland (Bogotá, Colombia) is a water system highly affected by the discharge of wastewater and activities developed by the urban matrix present in its surroundings. This study presents the design, construction and operation of a portable device for the simultaneous removal of solids and ammoniacal nitrogen from waters of the Córdoba wetland, which enables the increase of dissolved oxygen by means of gravity aeration. For that, it makes use of photovoltaic energy and incorporates a system of control of clogging filters and hose obstruction, in order to present an autonomous operation. Variables such as: loss of energy, diameter and density of filter materials, contact time, electricity consumption and weight, among others, were considered. All efficiency designs and tests were performed according to standardized standards. The dimensions of the device were estimated based on the hydraulic retention time. The results obtained allowed to measure the system efficiency, obtaining average removals of $74,4 \%$ and $89,5 \%$ for ammonia and total solids, respectively, and an increase in the concentration of dissolved oxygen up to $45 \%$, treating a flow rate of 14 $\mathrm{L} / \mathrm{min}$ and an autonomous working capacity of $3.5 \mathrm{~h}$. It is concluded that the device designed constitutes a promising alternative to improve the water quality of the Córdoba wetland at the in situ level, which can potentially be used for water treatment in other water systems with similar characteristics.
\end{abstract}

Keywords: Aeration, ammonium, control system, eutrophication, photovoltaic energy.

\section{INTRODUCCIÓN}

Los humedales constituyen uno de los entornos más productivos del mundo. Son cunas de diversidad biológica, fuente de agua y productividad primaria de los que innumerables especies animales y vegetales dependen para subsistir. Debido la potencial influencia de actividades antrópicas, estos sistemas se han visto seriamente amenazados y por tanto es prioritario generar estrategias para su preservación y restauración. (Guillot \& Pinilla, 2017).

Bajo adecuadas condiciones, los humedales ejercen un proceso de descontaminación de efluentes (industriales o domésticos). No obstante, fenómenos de sobrecarga, variaciones de flujo, factores climáticos y limitado retiro de escombros reduce considerablemente su estabilidad, lo cual imposibilita la degradación satisfactoria de agentes contaminantes presentes en el sistema (Rodríguez, 2017; Suaza et al, 2020). A pesar de esto, la información científica y técnica asociada al desarrollo de procesos de descontaminación de humedales, enfocados a la reducción simultánea de contaminantes específicos, es relativamente limitada.

En los últimos años se han venido incrementando los estudios referentes a la conservación y restauración de los humedales. La profundización de los conocimientos sobre el manejo ambiental de estos sistemas hídricos ha servido como base para el desarrollo de un número creciente de políticas asociadas a su gestión y manejo, (Martínez, Rodríguez, \& Hernández, 2014; Angulo et al, 2017).

El humedal Córdoba (Bogotá, Colombia), ha sido altamente afectado y transformado como consecuencia de la 
expansión del casco urbano y hábitos de los pobladores de la zona (Camargo, 2016; Sara-Lilia, 2019). Adicionalmente, el humedal presenta diversos problemas de saneamiento ambiental, dentro de los cuales se destaca el vertimiento de aguas residuales domésticas e industriales (Instituto de Estudios Ambientales, 2007).

Considerando el impacto ambiental al que ha estado expuesto el humedal Córdoba, y a la creciente necesidad de generar tecnologías económicamente viables que integren diversas operaciones de remediación de ambientes contaminados (alternas a procesos convencionales robustos y de alto costo como también que ataquen causas del problema que no se han abordan), en el presente artículo se planteó el desarrollo de un prototipo de dispositivo que opere de forma controlada, y que posibilite la mitigación de procesos de eutrofización en este tipo de sistemas. Para ello, se propuso diseñar un dispositivo basado en la remoción simultánea de parámetros de interés ambiental (Villamizar \& Justinico, 2017; Rivera et al, 2017; Melo et al, 2017).

\section{METODOLOGÍA}

\section{1 Área de estudio y colección de muestras}

El Humedal Córdoba se encuentra ubicado en la localidad de Suba (Bogotá, Colombia). Cuenta con una extensión de 40,5 ha y es alimentado por aguas de los canales "Molinos", "Callejas" y "Córdoba", los cuales constituyen afluentes altamente contaminados por vertimientos domésticos e industriales. (Fundación Humedales de Bogotá, 2013; Rojas, 2016; Secretaría Distrital de Ambiente de Bogotá, 2018).

Con la finalidad de realizar la configuración del sistema a emplear en el dispositivo, y las evaluaciones de su eficiencia, fueron seleccionados 4 puntos para colecta de muestras (Figura 1). En total fueron realizados 3 muestreos, colectando un volumen de $20 \mathrm{~L}$ por punto.
Las muestras fueron La caracterización fisicoquímica de las muestras fue realizada según los procedimientos establecidos en APHA (2005). Los parámetros de $\mathrm{pH}$ y oxígeno disuelto fueron determinados in situ empleando un medidor multiparámetro HANNA HI 991301 y oxímetro Az8403.

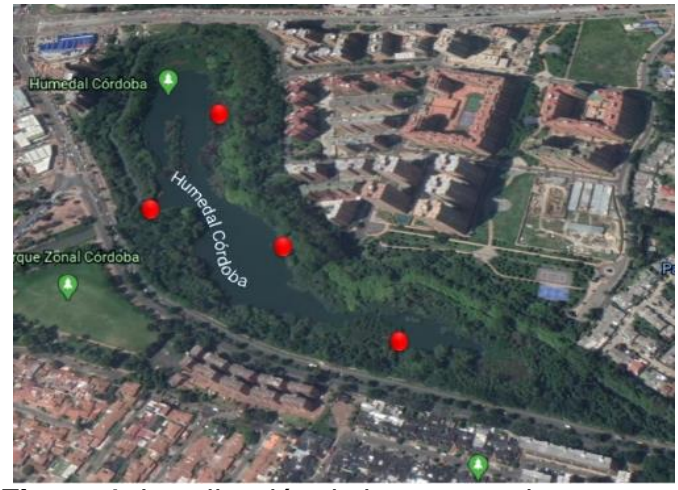

Figura 1. Localización de los puntos de muestreo en el humedal Córdoba. Fuente: Autor.

\subsection{Configuración del sistema de tratamiento}

Para realizar la configuración del sistema se procedió a determinar las características fisicoquímicas en uno de los puntos del humedal, con la finalidad de determinar los parámetros de calidad del agua de mayor relevancia a ser tratados por el dispositivo. Con dichos análisis fueron obtenidos los siguientes resultados: pH 6,4; Turbidez 371 NTU; Sólidos totales 1752 mg/L; Demanda química de oxígeno (DQO) $380 \mathrm{mg} / \mathrm{L}$; oxígeno disuelto (OD) $2,65 \mathrm{mg} / \mathrm{L}$; Nitratos $125 \mathrm{mg} / \mathrm{L}$ y amonio 4,00 $\mathrm{mg} / \mathrm{L}$.

A partir de la caracterización de las muestras de agua del humedal Córdoba, se determinó que el dispositivo debe ser particularmente eficiente en el aumento de la concentración de OD, la remoción de turbidez o SST, y la reducción de compuestos nitrogenados, particularmente amonio, dado que este compuesto puede ser precursor de otros compuestos nitrogenados que presentan alta concentración como por ejemplo nitratos, mediante nitrificación. 
Las etapas a considerar en el dispositivo, para el mejoramiento de la calidad del agua fueron:

Etapa 1: Filtro de grava para disminución de SST y la turbidez. (Metcalf \& Eddy, 2003).

Etapa 2: Enfocada en la disminución de SST y DQO particulada. Según Hamoda (2004) estas características disminuyen hasta un $99 \%$ con un filtro de arena.

Etapa 3: Adsorción de Amonio por medio de un filtro de Zeolita. Este compuesto presenta un intercambio iónico y una eficiencia entre 2,7-30,6 mg de $\mathrm{NH} 4 / \mathrm{g}$ Zeolita (Wang \& Peng, 2010).

Etapa 4: Enfocada en la reducción de olor y compuestos orgánicos volátiles por medio de adsorción con un filtro de carbón activado.

Finalmente se consideró el incremento de OD disuelto mediante oxigenación por gravedad.

\subsection{Parámetros de diseño del sistema de tratamiento}

Considerando lo anteriormente descrito, el diseño del sistema se basó en el desarrollo de un sistema de filtraciónadsorción de múltiple etapa. Para la oxigenación se optó por un aireador de bandeja múltiple que posibilite el contacto del agua con el aire por caída libre. El caudal de diseño a emplear (Qd) fue de 14 $\mathrm{L} / \mathrm{min}$. Según las disposiciones del RAS (2012) y los ejemplos de Romero (2013), para un sistema de aireación por bandejas se requiere una carga inferior a 100 $\mathrm{m}^{3} / \mathrm{m}^{2}$.d. Consecuentemente:

$$
\begin{gathered}
Q d=14 \mathrm{~L} / \mathrm{min} \\
Q d=20,16 \mathrm{~m}^{3} / \mathrm{d}
\end{gathered}
$$

La carga hidráulica superficial $(\mathrm{CH})$ fue establecida en $35 \mathrm{~m}^{3} / \mathrm{m}^{2}$.d. Por tanto, el área total necesaria $(\mathrm{At})$ estaría dada por:

$$
\begin{aligned}
& \text { At }=Q d / C H \\
& A t=0,57 \mathrm{~m}^{2}
\end{aligned}
$$

Como el sistema consta de 4 unidades $(n=4)$ entonces, el área de cada bandeja $(A b)$ es:

$$
\begin{gathered}
A b=A t / n \\
A b=0,14 m^{2}
\end{gathered}
$$

Para calcular el número de perforaciones previamente se debe conocer las dimensiones de las bandejas $(\mathrm{db})$, se hace el siguiente calculo:

$$
d b=\sqrt{A t / n}
$$

Las dimensiones de las bandejas fueron de 0,38 $\mathrm{m} \times 0,38 \mathrm{~m}$. Para el cálculo del número de perforaciones de las bandejas se adoptaron $\mathrm{N}$ orificios de $0,4 \mathrm{~cm}$ de diámetro (Do), teniendo en cuenta el tamaño promedio del granulo del material del lecho filtrante que se utilizará y un espacio entre orificio de $2,5 \mathrm{~cm}$ (Eo) recomendado por Romero (2012).

$$
\begin{aligned}
& N=\frac{d b-E o}{D o+E o} \\
& \mathrm{~N}=12,24 \approx 12
\end{aligned}
$$

Se usaron 12 filas de orificios cada uno de $4 \mathrm{~mm}$ separado entre si $2,5 \mathrm{~cm}$ con los orificios de borde a $3,0 \mathrm{~cm}$ de cada extremo. Para el montaje fueron empleadas bandejas rectangulares de 0,4 $\mathrm{m} \times 0,35 \mathrm{~m}\left(\mathrm{Ab}=0,14 \mathrm{~m}^{2}\right)$ atendiendo los parámetros de diseño. Para la separación entre las bandejas se adoptaron los criterios establecidos por el RAS (2012): $0,15 \mathrm{~m}$. Consecuentemente, el tiempo de contacto del dispositivo fue calculado mediante la siguiente expresión:

$$
t=n \sqrt{2 h / g}
$$

Donde, $\mathrm{t}=$ tiempo de exposición.

$\mathrm{h}=$ altura de cada bandeja.

$\mathrm{n}=$ Número de bandejas. $\mathrm{g}=$ Aceleración de la gravedad. 
El tiempo de exposición al aire del sistema fue estimado en $0,7 \mathrm{~s}$. Las características de los materiales filtrantes seleccionados se indican en la Tabla 1.

\subsection{Diseño del sistema electrónico}

En esta etapa se establecieron inicialmente los requerimientos del sistema eléctrico. Se diseñó un sistema de control con alarma que identifique cuando los filtros están colmatados y cuando se presenta una obstrucción en las mangueras que permiten la circulación del agua por el dispositivo. Por último, se seleccionaron los materiales según los requerimientos y se elaboró el esquemático electrónico y la programación del sistema de control.

En la Figura 2, se presenta el diagrama de bloques básico del prototipo dispositivo. Primero, se succiona el agua de humedal y se lleva a la parte superior del dispositivo, donde por gravedad pasa por los filtros hasta llegar de nuevo al cuerpo de agua. Adicionalmente, los filtros poseen sensores que determinan cuando estos estén colmatados o la presencia de algún objeto que impida el correcto funcionamiento del dispositivo y activan una alarma visual.

El sistema eléctrico-electrónico fue conformado por un sistema de alimentación eléctrica, la cual se realiza con un sistema fotovoltaico y un circuito de control que avisa al usuario la obstrucción de la manguera de succión del agua y la colmatación de los filtros, por medio de una alerta visual.

El diseño del circuito utilizado en el dispositivo se muestra en la Figura 3. En el circuito se utilizó un optoacoplador PC817, el cual permite encender y/o apagar la bomba de succión del agua cuando el Arduino le envía la señal al optoacoplador.

El diseño del software se realizó en un Arduino, el cual permite tener un control On-Off de la bomba de succión del agua, cuando esta se apaga se prende uno de los cinco leds del dispositivo. Cuatro leds corresponden a los sensores de nivel de las bandejas que avisan la colmatación de alguno de los filtros y el quinto led avisa la colmatación de la manguera de la bomba debido a una disminución del caudal, la cual determina por medio de un sensor de caudal.

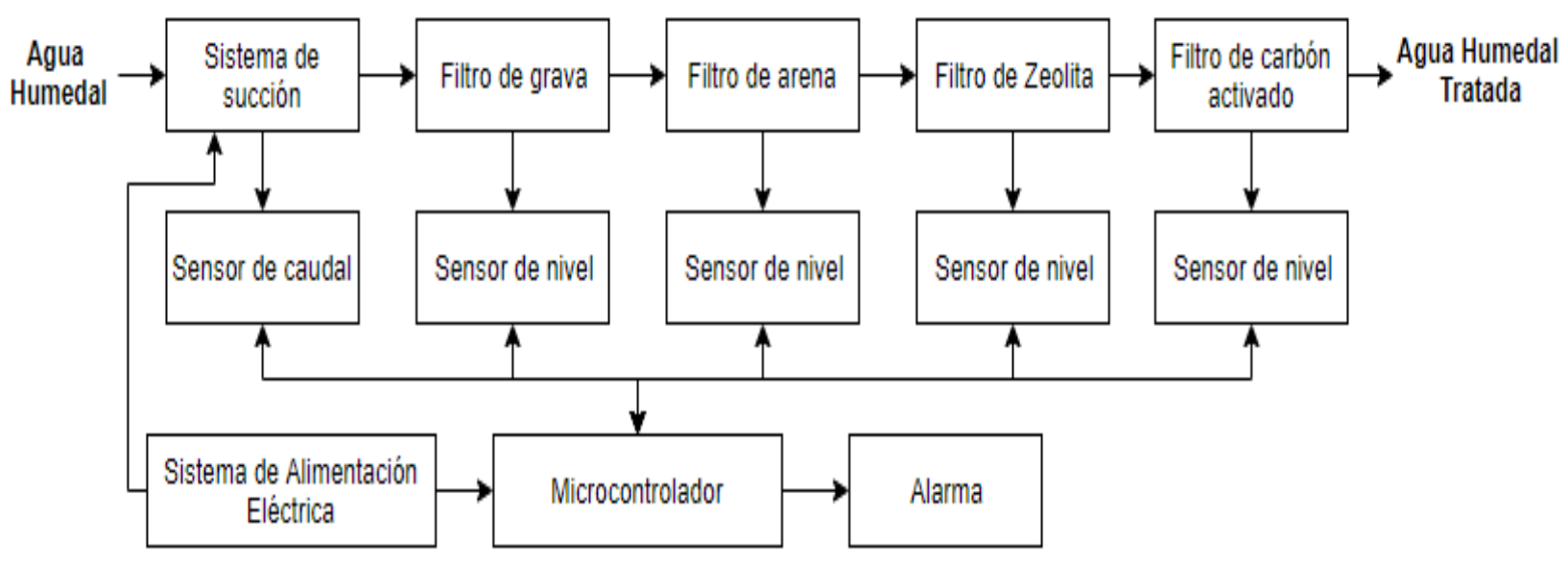

Figura 2. Funcionamiento del dispositivo. Fuente: Autor. 


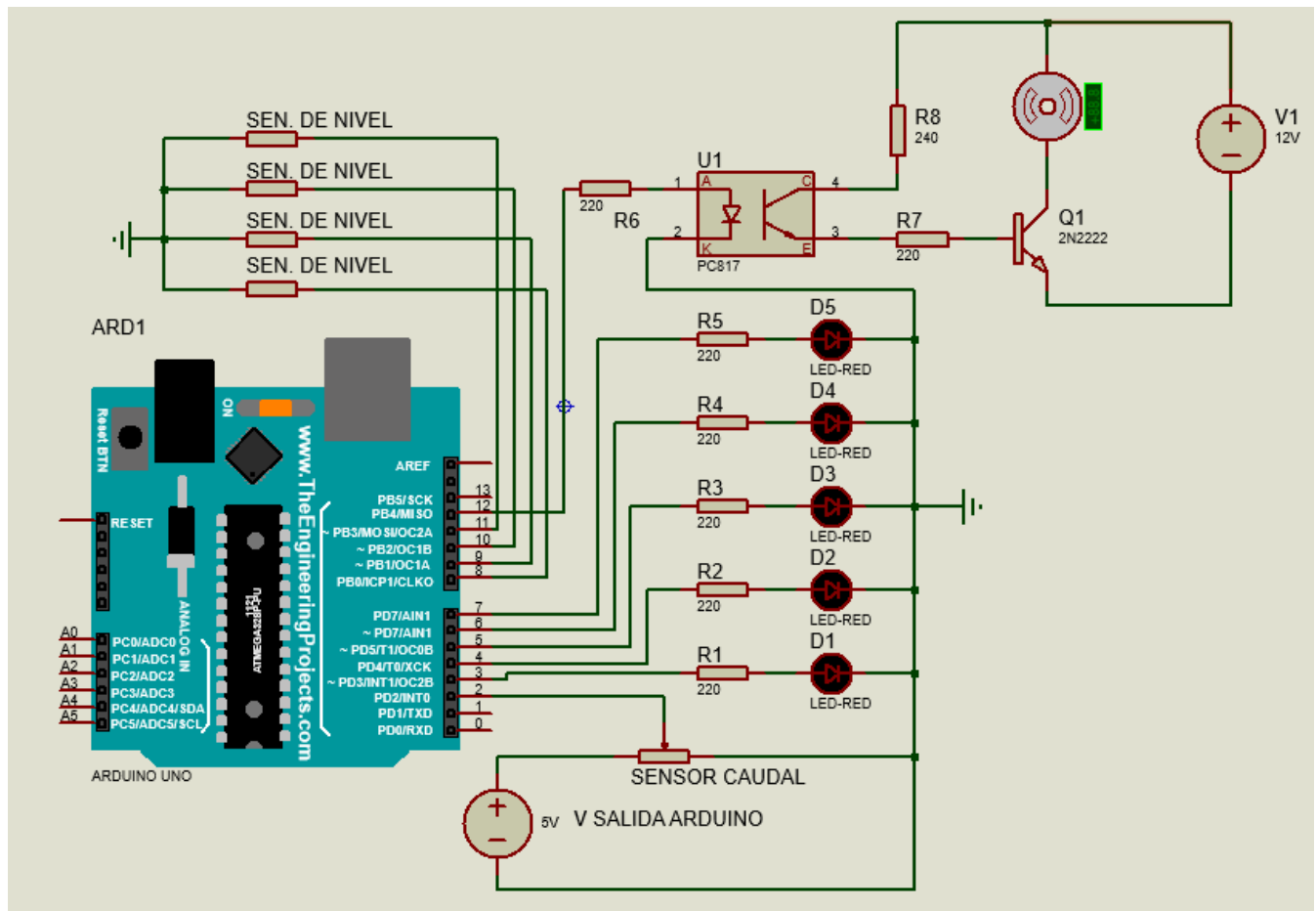

Figura 3. Circuito de control del dispositivo. Fuente: Autor.

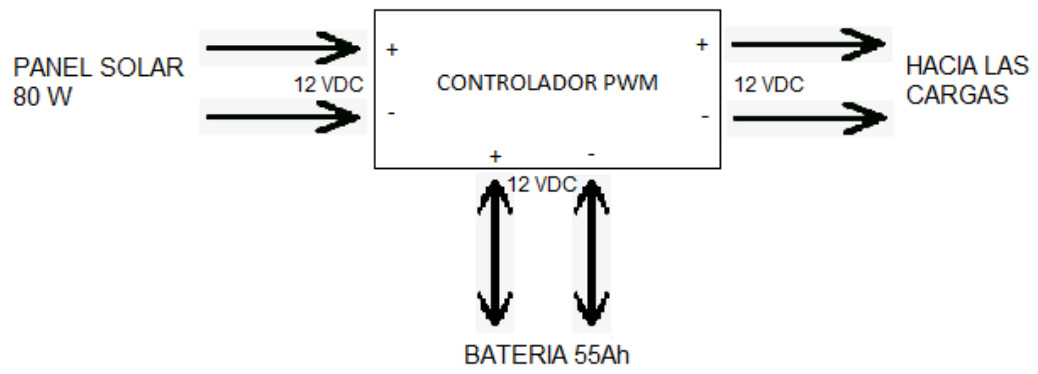

Figura 4. Sistema fotovoltaico. Fuente: Autor.

El circuito y la batería del sistema fotovoltaico se ubicaron en una caja de acrílico o en la parte superior del dispositivo y debajo del panel solar, con la finalidad de proteger los componentes de las condiciones climáticas. En la parte delantera se adaptaron leds de la alerta visual.

La Figura 4 se muestra el esquema del sistema fotovoltaico, el cual se implementó mediante un panel solar policristalino de 80 Watts conectado a un controlador PWM que evita sobrecargas y regula la carga de la batería de $12 \mathrm{~V}$, la cual tiene una capacidad de 55 Ah y que suple la energía requerida por las cargas.

Para el cálculo del consumo eléctrico del dispositivo fueron considerados los consumos de corriente nominales de cada componente así:

$\mathrm{W}=\mathrm{I} \times \mathrm{V} \times \mathrm{h}$

Donde:

$\mathrm{W}=$ Consumo del componente

I = Corriente nominal

$\mathrm{V}=$ Voltaje de funcionamiento 
$\mathrm{h}=$ Horas que va a funcionar

\section{ANÁLISIS Y DISCUSIÓN DE RESULTADOS}

\subsection{Sistema de filtración y aireación.}

El sistema para el mejoramiento de la calidad del agua se basó en el contacto del efluente con un lecho filtrante. En la Tabla 1, se muestran las características de los materiales seleccionados para realizar la filtración, la cantidad de material que se utilizó, la altura del lecho y el espacio que queda en cada bandeja. Toda la estructura fue elaborada el acero Cold Rolled con recubrimiento de pintura electrostática. EI material filtrante y la configuración de las bandejas empleadas se observan en la Figura 5.

Conforme lo establecido por Fair, Geyer, Okun y Ayanegui (1999) y Romero (2013), fue seleccionada una distancia entre bandejas de $12 \mathrm{~cm}$ para generar una óptima eficiencia en la aireación.

Tabla 1. Características de los materiales filtrantes seleccionados

\begin{tabular}{ccccc}
\hline Material Filtrante & $\begin{array}{c}\text { Diámetro del } \\
\text { poro }\end{array}$ & Cantidad & $\begin{array}{c}\text { Altura del lecho } \\
\text { filtrante }\end{array}$ & $\begin{array}{c}\text { Espacio entre el lecho filtrante y la } \\
\text { parte superior de la bandeja }\end{array}$ \\
\hline Grava & $4-6 \mathrm{~mm}$ & $18,5 \mathrm{~kg}$ & $8 \mathrm{~cm}$ & $5 \mathrm{~cm}$ \\
Arena & $1-4 \mathrm{~mm}$ & $15 \mathrm{~kg}$ & $8 \mathrm{~cm}$ & $5 \mathrm{~cm}$ \\
Zeolita & $1-4 \mathrm{~mm}$ & $7 \mathrm{~kg}$ & $5 \mathrm{~cm}$ & $8 \mathrm{~cm}$ \\
Carbón Activado & $1-4 \mathrm{~mm}$ & $7 \mathrm{~kg}$ & $7 \mathrm{~cm}$ & $6 \mathrm{~cm}$ \\
\hline
\end{tabular}

Fuente: Autor.

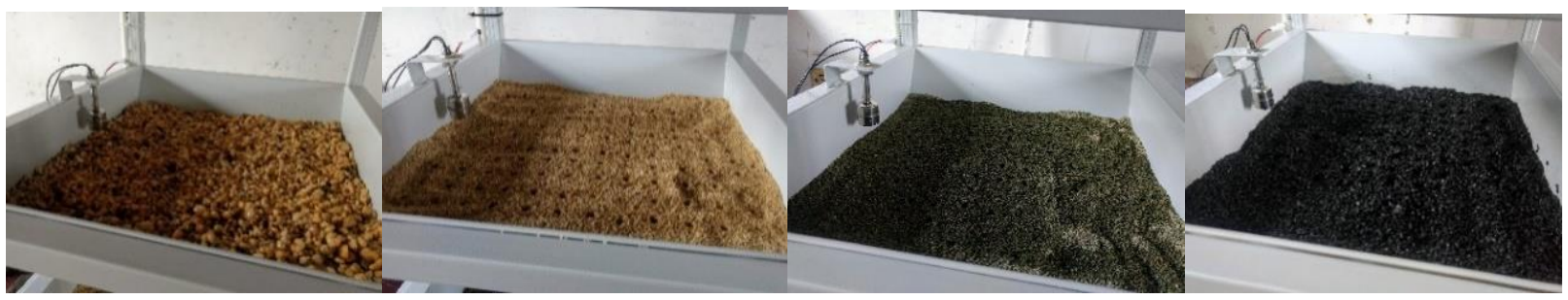

Figura 5. Material filtrante del dispositivo. De izquierda a derecha: Grava, arena, zeolita y carbón activado.

Fuente: Autor.

\subsection{Sistema eléctrico-electrónico.}

El sistema electrónico y la batería del sistema fotovoltaico se ubicaron dentro de una caja de acrílico, con la finalidad de protegerlo de las condiciones climáticas. Los ensayos preliminares permitieron evidenciar el suministro de energía del sistema fotovoltaico al sistema electrónico, para encender el Arduino y la bomba de succión del agua que se activa con la señal enviada por el microcontrolador. En caso de colmatación el sensor de nivel de agua se acciona al ascender el nivel del agua de la bandeja fuera de normal, se para el funcionamiento de la bomba y se enciende una señal lumínica por la parte posterior de la caja electrónica que indica que se debe retirar la bandeja con el filtro y hacer el correspondiente mantenimiento.

\subsection{Características operacionales}

Las características generales del dispositivo fueron las siguientes:

- Tamaño del dispositivo: $162 \mathrm{~cm}^{*} 65$ $\mathrm{cm}^{*} 55 \mathrm{~cm}$.

- Peso del dispositivo: 111,8 kg (Aprox. $140 \mathrm{~kg}$ peso con el agua).

- Cantidad de bandejas: 4 .

- Área de la bandeja: 0,14 m².

- Cantidad de perforaciones de la bandeja: 132 perforaciones.

- Distancia entre bandejas: $12 \mathrm{~cm}$. 
El dispositivo diseñado tiene la capacidad de funcionar autónomamente debido a que cuenta con un sistema de energía fotovoltaico. En la Tabla 2 se muestran los consumos calculados para todos los elementos electrónicos y eléctricos que conforman el sistema de control.
El tiempo de funcionamiento fue fijado a intervalos de $3,5 \mathrm{~h}$, considerando las características de operación de la bomba. El consumo eléctrico estimado se indica en la Tabla 2. En la Figura 6 se observa la configuración final del dispositivo.

Tabla 2. Consumo eléctrico del dispositivo.

\begin{tabular}{|c|c|c|c|c|}
\hline Componente & Consumo (W/h) & Cantidad & $\begin{array}{c}\text { Horas de } \\
\text { funcionamiento } \\
\text { (h) }\end{array}$ & $\begin{array}{c}\text { Total } \\
\text { Consumo } \\
\text { (W/h) }\end{array}$ \\
\hline Arduino uno & 0.55 (Prometec, 2019) & 1 & 3.5 & 1.9 \\
\hline Sensor de flujo YF-201 & 0.075 (Mantech Electronics, 2019) & 1 & 3.5 & 0.26 \\
\hline Sensor de nivel ES7510 & 2.5 (Amazon, 2019) & 4 & 3.5 & 8.75 \\
\hline Bomba de agua $14 \mathrm{~L} / \mathrm{min}$ & 14.4 (Electronilab, 2019) & 1 & 3.5 & 50.4 \\
\hline \multirow[t]{2}{*}{ Led } & 0.1 (Alibaba, 2019) & 5 & 3.5 & 0.35 \\
\hline & TOTAL & & & 61.6 \\
\hline
\end{tabular}

Fuente: Autor.

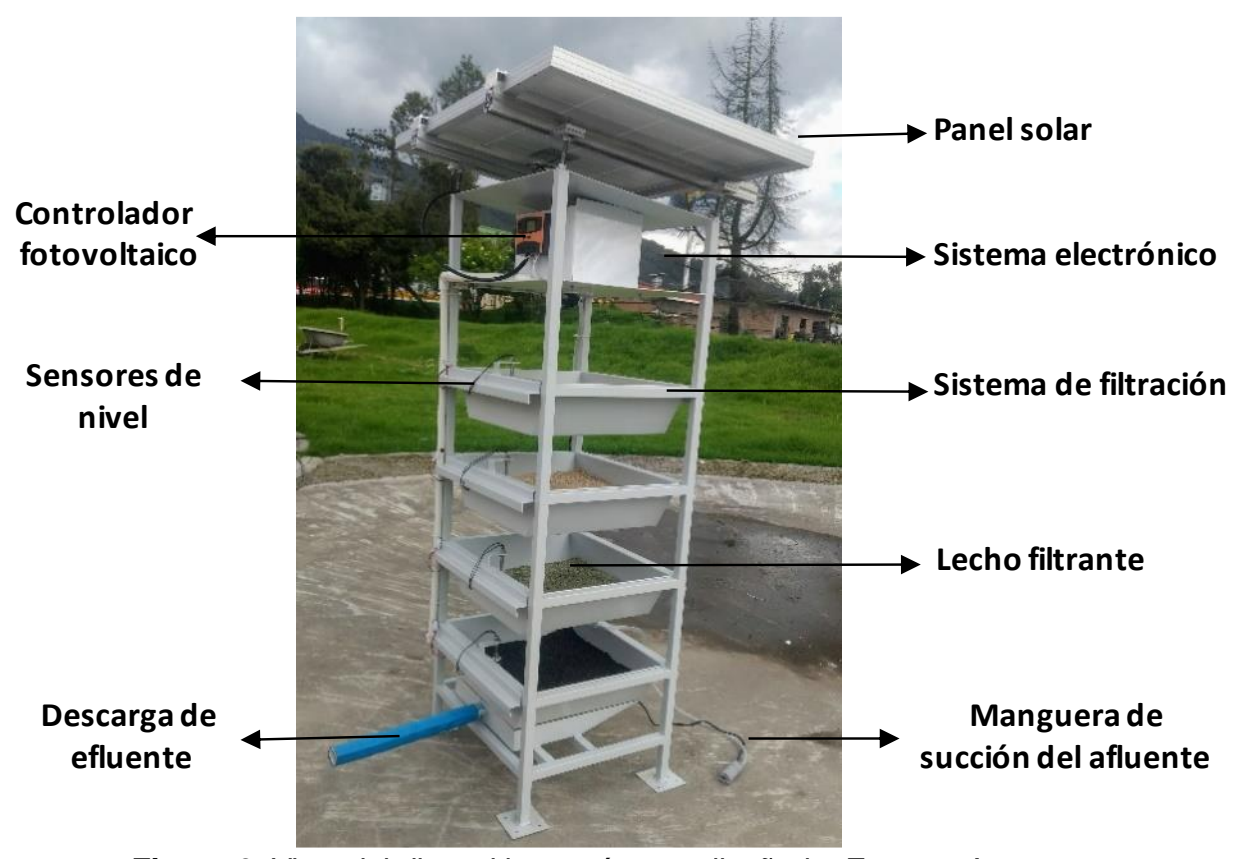

Figura 6. Vista del dispositivo autónomo diseñado. Fuente: Autor.

\subsection{Desempeño del dispositivo}

Para la evaluación del desempeño fueron colectadas y analizadas muestras del humedal, las cuales fueron sometidas al tratamiento con el dispositivo. El volumen total tratado por el mismo en cada corrida fue de $10 \mathrm{~L}$, provenientes de una muestra compuesta de los cuatro puntos seleccionados (80 L). En la Tabla 3 se muestran los resultados medios obtenidos de análisis por triplicado de los principales parámetros evaluados.

El coeficiente de variación de los resultados obtenidos fue inferior al $10 \%$. Fue evidenciado un porcentaje de remoción global para sólidos totales de $89,5 \%$. Para el caudal de trabajo (14 L/min.), este porcentaje evidencia una alta eficiencia del dispositivo en la remoción de este parámetro. Gualteros y Chacón Rodríguez (2015), empleando un filtro con lechos de arena fina y grava similar al de este estudio lograron remociones de 
solidos totales de entre el 99 y $100 \%$. No obstante, el caudal empleado en dicho estudio fue de 1,2 L/min.

La adsorción media de amonio fue del $74,4 \%$. Este resultado se puede contrastar con el trabajo realizado por Stefanakis y Tsihrintzis (2012) donde se logra el $78 \%$ de adsorción de amonio con zeolita en muestras de agua de humedal tratada a través de un filtro de flujo vertical, pero con un caudal menor a nuestro dispositivo y tiempos de retención en lecho filtrante mayores.

La remoción media global de DQO fue de $36 \%$. El bajo desempeño del dispositivo en relación a esta variable se debe al caudal de operación y la configuración del sistema. Remociones más altas de este parámetro pueden ser obtenidas con caudales más bajos o con el desarrollo de otro tipo de lechos filtrantes (Meneses, 2016; Jóźwiakowski K, 2017).

Por otra parte, se logró un aumento del oxígeno disuelto del $44,9 \%$ debido a la aireación del agua que se presenta de bandeja a bandeja. En contraste, el diseño de Cárdenas y Medina (2017) trabajando con aguas superficiales, obtuvo un incremento de un $29,37 \%$ de oxígeno disuelto con un caudal de $6 \mathrm{~L} / \mathrm{min}$. En consecuencia, el caudal tratado en este estudio influye de forma positiva para el incremento de este parámetro. Para las muestras 2 y 3 (Tabla 3) se logró llevar el oxígeno disuelto al valor establecido en la norma colombiana para la conservación de flora y fauna (Decreto 3930 de 2010), OD > $5 \mathrm{mg} / \mathrm{L}$.

En las muestras de agua tratadas se evidencia una disminución de la turbidez, debido fundamentalmente a que una alta concentración de los sólidos presentes se encuentra en suspensión. Una turbidez alta disminuye la producción de oxígeno y causa que la vegetación del fondo del cuerpo de agua no reciba la luz necesaria para realizar el proceso de la fotosíntesis, esto ocasiona una disminución del oxígeno disuelto, que a su vez produce un aumento en los fosfatos, nitritos y nitratos (Romero, 2013). Por tanto, esta característica de desempeño es fundamental para el mejoramiento de la calidad del agua del humedal.

Tabla 3. Resultados fisicoquímicos obtenidos en el afluente y efluente del dispositivo tratando aguas del humedal Córdoba.

\begin{tabular}{lcccccc}
\hline \multirow{2}{*}{ Parámetro } & \multicolumn{2}{c}{ Muestra 1 } & \multicolumn{2}{c}{ Muestra 2 } & \multicolumn{2}{c}{ Muestra 3 } \\
\cline { 2 - 7 } & Afluente & Efluente & Afluente & Efluente & Afluente & Efluente \\
\hline ST (mg/L) & $1752,3 \pm 620$ & $117 \pm 46$ & $2478 \pm 867$ & $368 \pm 148$ & $1289,7 \pm 294$ & $141 \pm 77,2$ \\
SST (mg/L) & $1318,3 \pm 195$ & $131 \pm 21,1$ & $3010,7 \pm 172,2$ & $118 \pm 12,5$ & $1255,7 \pm 86,2$ & $203,3 \pm 26,6$ \\
SSD (mL/L) & $19 \pm 0,01$ & $0,3 \pm 0,01$ & $43,5 \pm 9,19$ & $0,65 \pm 0,07$ & $17,5 \pm 0,71$ & $0,35 \pm 0,07$ \\
OD (mg/L) & $2,65 \pm 0,21$ & $4,65 \pm 0,21$ & $4,45 \pm 0,07$ & $5,8 \pm 0,14$ & $4,2 \pm 0,28$ & $5,4 \pm 0,28$ \\
Nitratos (mg/L) & $125 \pm 5,0$ & $100 \pm 1,0$ & $100 \pm 1,0$ & $75 \pm 0,5$ & $100 \pm 2,0$ & $75 \pm 0,5$ \\
Amonio (mg/L) & $2,5 \pm 0,71$ & $0,75 \pm 0,35$ & $4 \pm 1,4$ & $0,75 \pm 0,35$ & $2,5 \pm 0,71$ & $0,75 \pm 0,35$ \\
DQO (mg/L) & $567,5 \pm 14,8$ & $249,5 \pm 58,7$ & $180,5 \pm 12,0$ & $102,6 \pm 20,4$ & $314 \pm 52,3$ & $288,7 \pm 67,0$ \\
pH & 6,37 & 6,24 & 7,93 & 6,44 & 6,21 & 6,12 \\
Turbidez (NTU) & 371 & \multicolumn{2}{c}{52,8} & 892 & 55,2 & 185 \\
ST: Sólidos totales; SST: Sólidos suspendidos totales; SSD: Sólidos sedimentables; OD: Oxígeno disuelto; DQO: Demanda química de oxígeno.
\end{tabular}

\section{Conclusiones}

Se diseñó un sistema en columna de bandejas perforadas para la filtración rápida de sólidos, adsorción de amonio y aireación por gravedad de aguas del humedal Córdoba. El dispositivo cuenta con un sistema electrónico que posibilita la detección y control de colmatación de filtros y obstrucción en la manguera, el cual 
obtiene su suministro eléctrico de un sistema fotovoltaico aislado. El dispositivo evidenció una alta eficiencia para el mejoramiento de la calidad del agua del humedal Córdoba, obteniendo un aumento medio de oxígeno disuelto hasta de $45 \%$ (de 3.9 a $5.4 \mathrm{mg} / \mathrm{L}$ ), y una remoción de $74,4 \%$ y $89,5 \%$ para amonio y sólidos totales, respectivamente. El dispositivo diseñado constituye una alternativa promisoria para mejorar la calidad de las aguas del humedal Córdoba a nivel in situ y su concepto puede ser potencialmente empleado y optimizado para el tratamiento de aguas en otros sistemas hídricos similares características.

\section{Referencias Bibliograficas}

APHA. (2005). WEF, 2005. Standard methods for the examination of water and wastewater, 21, 258-259.

Angulo, W. J., Mendoza, J. A. y Uriel, H. U. (2017). Análisis de la vulnerabilidad por fenómenos de remoción en masa en la Cuenca Tanauca estudio de caso. Revista Ambiental Agua, Aire y Suelo. ISSN 19009178. Volumen (8), Numero (2). DOI: https://doi.org/10.24054/1900 9178.v2.n2.2017.3276

Camargo, W. C. (2016). Modelación hidrologico-hidraulica de eventos de inundacion en el Rio Bogotá (sector tocanzipa-chia) usando HEC-RAS. Revista Ambiental Agua, Aire y Suelo. ISSN 1900-9178. Volumen (7), Numero (2). DOI: https://doi.org/10.24054/1900 9178.v2.n2.2016.3267

Cárdenas, G., Sánchez, I., Maya, J. y Solarte,A. (2015). Remoción de sólidos en aguas residuales de producción intensiva de trucha en un sistema de recirculación cerrado. (2015). Revista UNIMAR, 33(1), 229-236.

Cárdenas, A., y J. Medina. (2017). Diseño y Construcción de Una Planta de Tratamiento de Agua Potable a Escala Para El Laboratorio de
Hidráulica de La Universidad Santo Tomás. Universidad Santo Tomás.

Fair, G., Geyer, J. C., Okun, D. A., \& Ayanegui, J. S. (1999). Ingeniería sanitaria y de aguas residuales $\mathrm{Vol}$ 2. México: Limusa.

Fundación Humedales Bogotá (2013). El canal ecológico del Humedal Córdoba. Obtenido de Fundación Humedales

de Bogotá:http://humedalesbogota.co m/2013/03/18/el-caudal-ecologicodel-humedal cordoba/

Gualteros Díaz, L. J., \& Chacón Rodríguez, M. A. (2015). Estudio de la eficiencia de lechos filtrantes para la potabilización de agua proveniente de la quebrada la despensa en el municipio Guaduas Cundinamarca vereda la Yerbabuena. Universidad de la Salle.

Guillot, G., \& Pinilla, A. (2017). Estudios ecológicos en humedales de Bogotá: aplicaciones para su evaluación, seguimiento y manejo. Bogotá: Universidad Nacional de Colombia.

Hamoda, M. F., I. Al-Ghusain, and N. Z. ALMutairi. 2004. "Sand Filtration of Wastewater for Tertiary Treatment and Water Reuse." Desalination 164(3): 203-11.

Instituto de Estudios Ambientales. (Diciembre de 2007). Plan de manejo ambiental del humedal Córdoba: Problemática, valoración y evaluación. Obtenido de Ambiente Bogotá:

Jóźwiakowski, K. (2017). Efficiency of organic substance removal in a hybrid sand filter with horizontal flow. Journal of Water and Land Development, 35(1), 95-100.

Martínez, A. A., Rodríguez, J., \& Hernández, A. (2014). The wetlands landscapes. Conceptual and methodological aspects. MERCATOR, 13(2), 169-191.

Melo, J., Saavedra, S. y Ramón, J. A. (2017). Evaluación de la adsorcion 
de CU+2 y azul de metileno en biosorbentes de bajo costo obtenidos a partir de biomasa residual de la agroindustria de cítricos. Revista Ambiental Agua, Aire y Suelo. ISSN 1900-9178. Volumen (8), Numero (2). DOI: https://doi.org/10.24054/1900 9178.v2.n2.2017.3277

Meneses, V. B., Álzate, D. y Mosquera, J. (2016). Sistema de optimización de las técnicas de planificación en agricultura de precisión por medio de drones. Revista Ambiental Agua, Aire y Suelo. ISSN 1900-9178. Volumen (7), Numero (2). DOI: https://doi.org/10.24054/1900 9178.v2.n2.2016.3268

Metcalf\&Eddy. 2003. Wastewater Engineering: Treatment and Reuse, Fourth Ed. McGraw-Hill, Boston, USA. 4th ed. eds. Franklin L Burton, $\mathrm{H}$ David Stensel, and George Tchobanoglous. Boston: McGraw Hill.

Reglamento Técnico del sector agua Potable y Saneamiento Básico, RAS (2012). C. y. Ministerio de Vivienda, Titulo F, Sistemas de Aseo Urbano.

Rivera, H. U., Castellanos, C. y lbarra, A. (2017). Caracterización y cuantificación de los residuos sólidos realizado en el municipio de pamplona, $\mathrm{N}$ de $\mathrm{S}$, Colombia. Revista Ambiental Agua, Aire y Suelo. ISSN 1900-9178. Volumen (8), Numero

DOI: https://doi.org/10.24054/1900 9178.v2.n2.2017.3280

Rodríguez, Y. A. (2017). Conservación de humedales en el marco de gestión de cuencas hidrográficas. Puerto Rondón-Arauca. Revista Ambiental Agua, Aire y Suelo. ISSN 19009178. Volumen (8), Numero (2). DOI: https://doi.org/10.24054/1900 9178.v2.n2.2017.3281

Rojas, J. S. (2016). Aplicación del modelo estocástico WIENER-FPK en caudales medios del rio fonce en condiciones del sistema de Pearson. Revista Ambiental Agua, Aire y Suelo. ISSN 1900-9178. Volumen (7), Numero (1). DOI: https://doi.org/10.24054/1900 9178.v1.n1.2016.3256

Romero, J. A (2013). Tratamiento de aguas residuales: Teoría y principios de diseño. Bogotá: Editorial Escuela Colombiana de Ingeniería.

Sara-Lilia, Á. D. N., Sandra-Mónica, E. T., Lina-Marcela, C. C., XiomaraMichel, C. C., \& Wendy-Lizeth, R. A. (2019). Diagnosis of the bacteriological quality of the water of the Córdoba Wetland, Bogotá. Nova, 17(31), 87-95.

Secretaría Distrital de Ambiente de Bogotá. (2018). Humedales de Bogotá. Obtenido de Secretaría Distrital de Ambiente de Bogotá: http://humedalesdebogota.ambient ebogota.gov.co/inicio/\#

Stefanakis, A. I., \& Tsihrintzis, V. A. (2012). Use of zeolite and bauxite as filter media treating the effluent of Vertical Flow Constructed Wetlands. Microporous and Mesoporous Materials, 155, 106116.

Suaza, D. S., \& Quijano-Abril, M. A. (2020). Análisis multitemporal y caracterización de la vegetación hidrófita y helófita de un cinturón de humedales urbanos en el altiplano del Oriente antioqueño. Revista de la Academia Colombiana de Ciencias Exactas, Físicas y Naturales, 44(171), 639-651.

Villamizar, V. A. y Justinico, A. J. (2017). Reconstrucción paleoclimatica y paleoambiental de los territorios de la llanura inundable del araucana a partir del análisis de sedimentos recientes, Departamento de Arauca. Revista Ambiental Agua, Aire y Suelo. ISSN 1900-9178. Volumen (8), Numero

(1). 
A

DOI: https://doi.org/10.24054/1900

9178.v1.n1.2017.3272

Wang, Shaobin, \& Yuelian Peng. 2010. "Natural Zeolites as Effective Adsorbents in Water and Wastewater Treatment." Chemical Engineering Journal 156(1): 11-24. 